\title{
Complex response of an oscillating vertical cantilever with clearance
}

\author{
Krystian Lygas · Piotr Wolszczak • Grzegorz Litak $(\mathbb{D})$ Pawel Sțaczek
}

Received: 7 June 2018/Accepted: 29 July 2019/Published online: 8 October 2019

(C) The Author(s) 2019

\begin{abstract}
We study the dynamics of an elastic inverted pendulum with amplitude limiters excited horizontally. This particular model corresponds to a class of systems where a clearance is present naturally as an effect of imperfect clamping or it is included to tailor the response. We explore the complex responses of the system for a fixed value of amplitude clearance. The simulation and experimental results are analysed by a $0-1$ test, Fourier, and wavelet transforms. The results show that the system can vibrate with subharmonic solution where the main response frequency of a flexible beam is 3 times lower than the excitaion frequency. We claim that an inverted pendulum with imperfect clamping of mechanical resonator can be used in broad frequency band energy harvesting.
\end{abstract}

Keywords Nonlinear vibration ·

Intermittent response - Escape from a potential well · Energy harvesting

K. Łygas · P. Wolszczak · G. Litak ( $₫) \cdot$ P. Sta̧czek Department of Automation, Lublin University of Technology, Nadbystrzycka 36, PL-20-618 Lublin, Poland

e-mail: g.litak@pollub.pl

\section{Introduction}

Energy harvesting from ambient mechanical vibration is a competitive concept in order to ensure effective powering of small devices [1-4]. In particular, sensors or modern electronic applications can be designed to work directly on harvested energy, or indirectly powered by batteries with an energy recharging option using energy harvesting [1]. Earlier results were obtained by using a simple cantilever beams with piezoelectric, electrostatic, or electromagnetic couplings [5] working in a linear mode. In such a case the only excitation frequency coinciding to the natural frequency of the mechanical resonator is useful. It leads to significant limitations of the energy harvesting conditions based on linear resonance of the device [5, 6]. In other words, the energy harvesting device must be adjusted to the ambient energy sources and frequency available. Consequently, because of available frequencies exceeds the narrow region of the resonance, energy from variable sources of vibrations cannot be transduced effectively to the electrical power output. Some possibilities of improvements were indicated in increasing of additional mechanical damping to reach the impedance matching. It leads to broaden the resonance conditions to small bands around the resonance but simultaneously lowered the energetic efficiency [5, 6]. In parallel, the efficiency of energy harvesting were improved considerably by the 
application of synchronized switcher in the electric circuit $[7,8]$.

Recently, a number of non-linear devices have been proposed to maximise harvested energy over a wide range of excitation frequencies [9]. One of the important direction of development was the application of bistable or multistable mechanical resonators [10-14]. Appearance of inclined nonlinear resonance backbone curves, introducing multiple solutions with additional nonlinear resonances guaranteed broadening of the frequency bands in nonlinear resonators. This direction opened an development of application vibrational energy harvesting to the sources of variable amplitude and frequency, to modulated vibrations, vibrations with random components and non-ideal sources of vibrations [15-18].

Among various proposals for energy harvesting, inverted pendulum systems have been tested [19-22]. On the other hand, studies on horizontal beams with amplitude limiters can be also found [23]. In this paper we propose the mechanical resonator designed as a hinged inverted elastic pendulum with amplitude stoppers (Fig. 1). This could be considered as an imperfect clamped case comparing to the previous model characterized by regular clamping [19]. The aim is to test it for possible broadband response in the bending motion of higher frequency. Transverse bending motion can transform the vibrational energy into the electrical power [5]. Here, we focused on the subharmonic resonance in respect to the larger amplitude solution associated with the hopping between the potential wells.

\section{Modelling}

Our model (Fig. 1) is based on a vertical flexible beam with a tip mas excited horizontally (which can be found in [19]). However, instead of the usual clamping we consider the hinged connection with additional clearance. Such a system posses two potential wells. An additional nonlinear effect is related to impacts into the amplitude limiters. Similar flexible nonlinear beam systems were discussed in the papers [24-26]. Tang and Ren [24] and Xu and Yu [25] invented a control algorithm to stabilize the pendulum in the upright position. While Semenov et al. [26] provided the discussion of an inverted Kapica like pendulum with the vertical excitation. This pendulum was additionally exposed on backlash in the hysteretic switching coupling. In the subsequent papers Semenov at al. [27, 28] considered the horizontal excitation with a similar coupling between the excitation frame and the inverted pendulum. Their model is similar to our proposal [29], however we realize the clearance between amplitude limiters as (a)

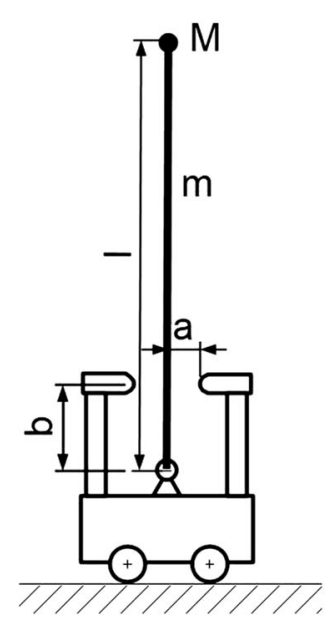

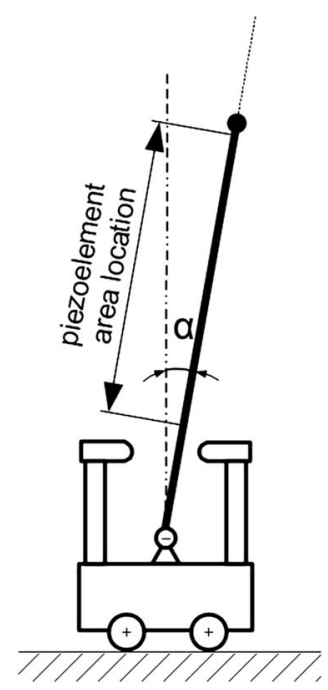

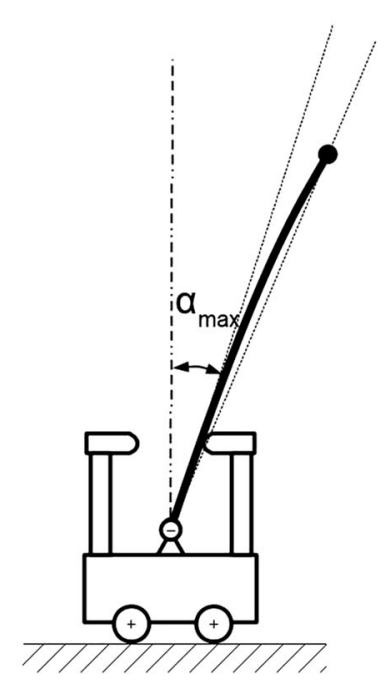

(b)

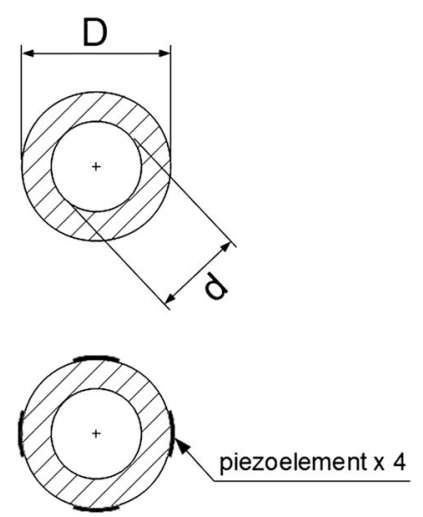

Fig. 1 Schematic picture of the elastic inverted pendulum with amplitude stoppers. The system dynamics is limited to planar motion. Note, the positions where piezoelectric patches can be places 
well as the contact between amplitude limiters and the pendulum in a different way. The possible placement of the piezoelectric is shown in Fig. 1b.

\subsection{Numerical model and simulation results}

In our multibody model we start with the distretization on $N=17$ mass elements and $N+1$ nodes along the beam length. Then the kinetic $T$ and potential $V$ energies can be expressed by $2 N$ degrees of freedom system (Fig. 2a):

$$
\begin{aligned}
& T=\sum_{i=1}^{N} \frac{\Delta m_{i}\left(\mathbf{v}_{i}+\mathbf{v}_{i-1}\right)^{2}}{8}+\sum_{i=1} \frac{\Delta I_{i} \dot{\alpha}_{i}^{2}}{2}+\frac{M \mathbf{v}_{N}^{2}}{2}, \\
& V=\sum_{i=1}^{N} \Delta m_{i} g \frac{\left(y_{i}+y_{i-1}\right)}{2}+\sum_{i=1}^{N} \frac{k\left(\alpha_{i}-\alpha_{i-1}\right)^{2}}{2}+M g y_{N},
\end{aligned}
$$

where $g=9.81 \mathrm{~m} / \mathrm{s}^{2}$ is the gravity acceleration, $M$ is a tip mass attached to the end node of No. $N, \mathbf{v}_{i}=\left[x_{i}, y_{i}\right]$ are vectors denoting local connecting node velocities, $\alpha$ are angles between the neighbour short beam elements. All the elements have the same shape and sizes. Note that $\alpha_{0}=0$ and the assumed $v_{0}=\dot{x}_{0}$ of harmonic motion in the horizontal direction:
$x_{0}=A \sin (\omega t), \quad y_{0}=0$,

where $A$ is the amplitude while $\omega$ is the corresponding frequency of kinematic excitation. Furthermore, $\Delta m_{i}=\Delta m$ is mass of a short beam element while $\Delta I_{i}=\Delta I$ is the moment of inertia for defined elements. Finally, $k$ is the local spring coefficient.

The Lagrangian of the beam without further constraints (limiters) has the following form:

$$
\begin{aligned}
& L\left(q_{1}, \ldots, q_{i}, \ldots, q_{N}, \dot{q}_{1}, \ldots, \dot{q}_{i}, \ldots, \dot{q}_{N}, t\right) \\
& =T\left(q_{1}, \ldots, q_{i}, \ldots, q_{N}, \dot{q}_{1}, \ldots, \dot{q}_{i}, \ldots, \dot{q}_{N}, t\right) \\
& \quad-V\left(q_{1}, \ldots, q_{i}, \ldots, q_{N}, \dot{q}_{1}, \ldots, \dot{q}_{i}, \ldots, \dot{q}_{N}, t\right),
\end{aligned}
$$

where the generalized coordinates this multistage inverted pendulum $q_{i} \in\left\{\alpha_{1}, \ldots, \alpha_{n}\right\}$ and their corresponding velocities $\dot{q}_{i} \in\left\{\dot{\alpha}_{1}, \ldots, \dot{\alpha}_{n}\right\}$. Consequently, the general equation of motions for such a kinetically forced inverted pendulum can be written as:

$\frac{d}{d t}\left(\frac{\partial L}{\partial \dot{q}_{j}}\right)-\frac{\partial L}{\partial q_{j}}=Q_{j}+Q_{n}^{i m p}$.

The generalized forces $Q_{j}$ appearing in the above equation are defined

$$
Q_{j}=-\beta\left(\dot{\alpha}_{j}-\dot{\alpha}_{j-1}\right)-\beta\left(\dot{\alpha}_{j}-\dot{\alpha}_{j+1}\right),
$$

while the additional generalized force $Q_{n}^{i m p}$
Fig. 2 a Schema of multibody model of the beam (divided on 17 equal small rigid beams) with limiters. Circles show the beam discretization. b The simplified flexible beam model as a three body model. Here $m=\Delta m$ is the mass of single small rigid beam (a)

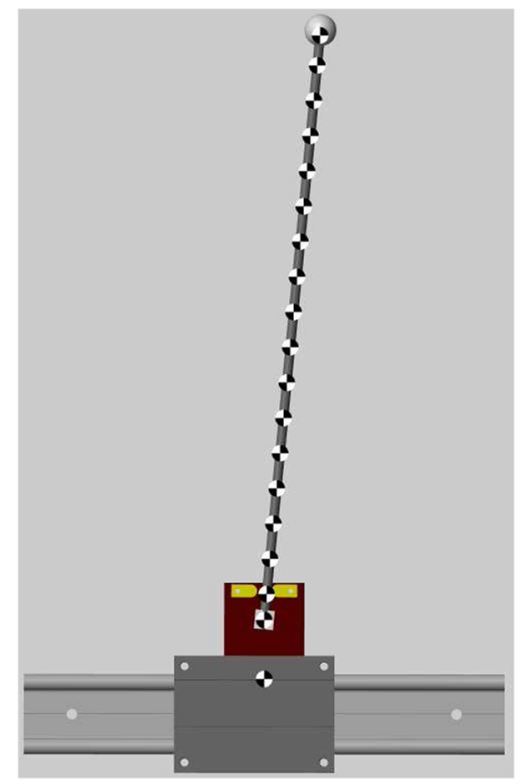

(b)

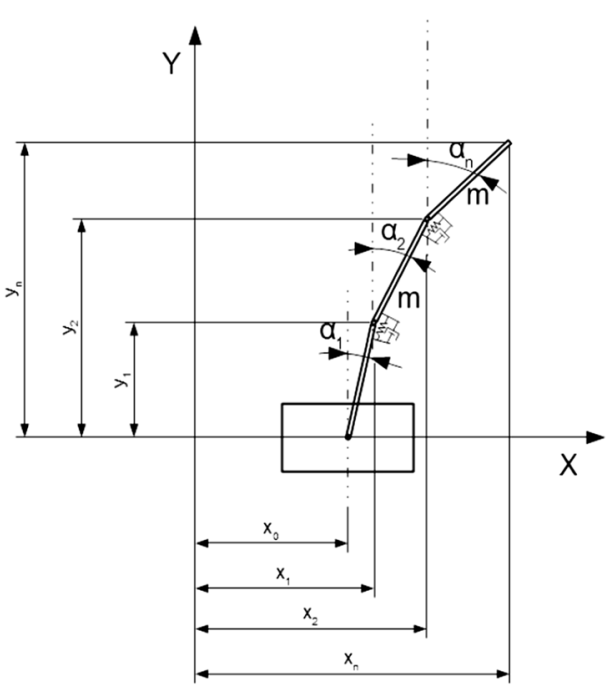


Table 1 Material and geometrical properties of beam and piezoelectric component

\begin{tabular}{ll}
\hline Length of the beam & $375 \mathrm{~mm}$ \\
Material density & $7850 \mathrm{~kg} / \mathrm{m}^{3}$ \\
Modulus of elasticity & $2 \times 10^{11} \mathrm{~Pa}$ \\
Shear modulus & $77 \mathrm{GPa}$ \\
Damping constant & 0.35 \\
Diameters of beam correction & $d=3.5 \mathrm{~mm}$, and \\
(hollow cylinder shape) & $D=6 \mathrm{~mm}$ \\
Tip mas & $11.95 \mathrm{~g}$ \\
Beam mas & $55.25 \mathrm{~g}$ \\
Contact stiffness & $10^{4} \mathrm{~N} / \mathrm{m}$ \\
Contact damping & $10^{2} \mathrm{~N} / \mathrm{m}$ \\
\hline
\end{tabular}

$Q_{n}^{i m p}=-\beta_{i m p} \dot{q}_{n}^{\perp}+\alpha_{i m p} q_{n}^{\perp}$,

is caused by an inelastic contact to one of amplitude limiters with local contact elasticity and damping properties (see Table 1 for parameter values and Figs. 1 and 2a for view). $q_{n}^{\perp}$ and $\dot{q}_{n}^{\perp}$ are average displacement and velocity perpendicular vector elements at corresponding beam element and the impact contact place. For simplicity, we neglected the torque on impacting beam elements. The limiters are attached to the beam elements depending on deflection shape and cause the whole beam to bounce.

The calculations of the dynamical response of the introduced pendulum-impact model were performed by using Maltab-Simulink and Simscape Multibody with Flexible Beam and Contact Forces libraries. Note that the partition on the 17 equal parts is a sufficient assumption for this software procedure.

In the system we have several important frequencies: the natural frequency of the beam (about $30 \mathrm{~Hz}$ and larger), excitation frequency and the characteristic frequency of the beam which is moving between impacting bumpers in the undeformed shape. Our intention is to provide off resonance solution in the studied system.

The results of simulations for chosen three values of the frequency $(f=10 \mathrm{~Hz}, 8 \mathrm{~Hz}$, and $5 \mathrm{~Hz})$ are presented in Figs. 3-5. These initial results were obtained for the first $30 \mathrm{~s}$. We show the displacement of trolley, holder angle, together with the Fourier spectrum (two lower panels of Fig. 3).
Note that after nonperiodic transient, vibrations are fairly regular as visible in the Fourier spectra. The trolley moves initially in a harmonic way, while the holder rotates irregularly. This is due to the nonlinearities in the system mainly double well potential and impacts of the pendulum to bumpers (amplitude limiters). For better clarity we show also additional parameter distance between the end points of the pendulum-a chord. Its variability in the time can also give a certain guidance about the beam deflection (see chord reduction in upper panels of Fig. 3) and possible effect on possible piezoelectric energy harvesting.

In Figs. 4 and 5 we present the results for the forcing frequency $f=8 \mathrm{~Hz}$ and $5 \mathrm{~Hz}$, respectively. At the first overview of the time series solutions of (frequency, $f$, and amplitude $A$ ) $f=8 \mathrm{~Hz}, A=9 \mathrm{~mm}$ and $f=5 \mathrm{~Hz}, A=9 \mathrm{~mm}$ in (Figs. $4 \mathrm{a}$ and $5 \mathrm{a}$ ), are irregular in the whole simulated time interval while the case of $f=5 \mathrm{~Hz}, A=9 \mathrm{~mm}$ (Fig. $5 \mathrm{~b}$ ) is periodic with a single frequency response. Other studied cases seam to be regular after a some transients. The Fourier spectra confirm these initial conclusions indicating larger low frequency bands in Figs. 3a and $4 \mathrm{a}$ for the angular displacement of the pendulum holder. Figure $4 \mathrm{a}$ shows the broad peak in the one third of the excitation frequency $f=2.7 \mathrm{~Hz}$. This is the frequency of the first mode large amplitude deflection oscillation of the beam-and-tip-mas system coupled to the pendulum holder. Interestingly, the results show that the pendulum converts the excitation signal to almost harmonic response of pendulum. This frequency is switched to one halve for higher excitation amplitude (Fig. 4b).

Furthermore, the chord reduction is well synchronized with the holder angle displacement and its time series. The corresponding Fourier spectra clearly show that the chord reduction happens two times (presumably in the left and right directions) during the single period of excitation. In addition to expected frequency doubling, the chord reductions in have broader character. It can be explained by overlapping of the bending oscillations originated from the impact interactions and coexistence of higher modes of oscillations, or their sub- and super-harmonics discussed in the previous studies [29]. 
(a)
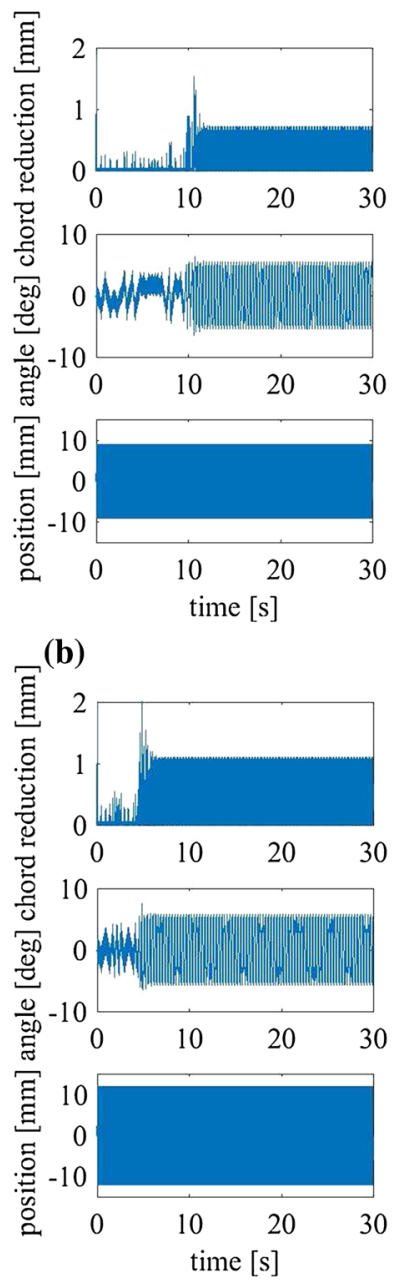
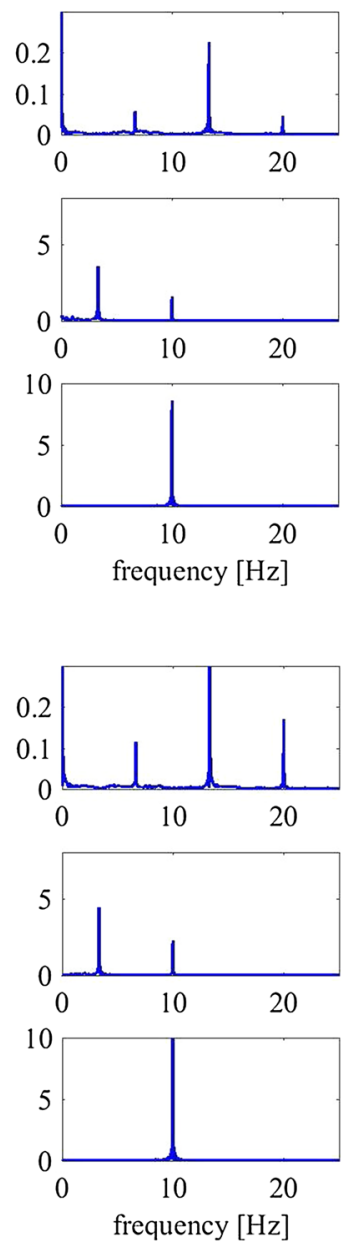

Fig. 3 Simulation results, the frequency and amplitude of kinematic excitation used: $f=10 \mathrm{~Hz}, A=9 \mathrm{~mm}$ (a) and $12 \mathrm{~mm}$ (b)

\subsection{Chaotic solution identification by using $0-1$ test}

The ' $0-1$ test' was invented by Gottwald and Melbourne [30, 31]. It can be applied to any system of a finite dimension to identify the chaotic dynamics. In analogy to stochastic systems, it is based on the statistical properties of the dynamical response. It is possible to quantify the response where only one parameter simulated, or measured in time can distinguish a chaotic system from a regular one (see ref. $[32,33]$ for overview). Namely, the control parameter (to be defined in this section) $K$ takes values 1 and 0 (a)
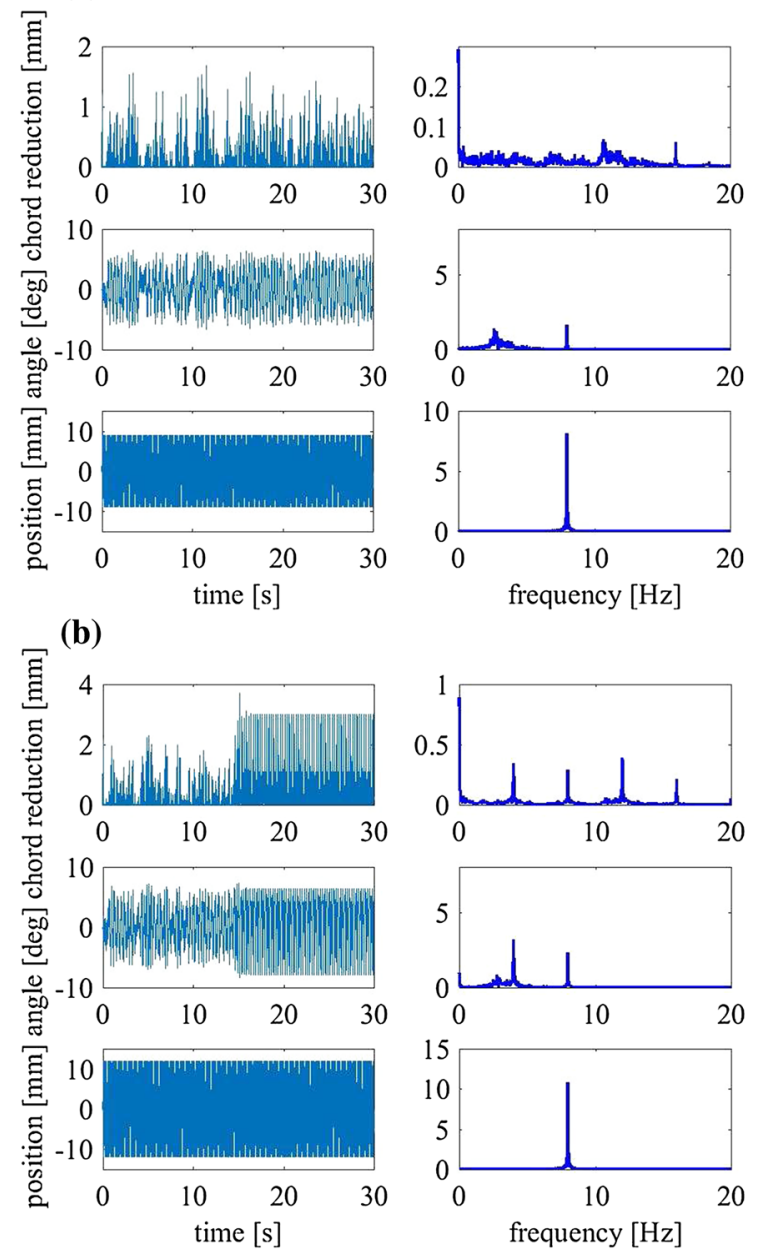

Fig. 4 Simulation results, the frequency and amplitude of kinematic excitation used: $f=8 \mathrm{~Hz}, A=9 \mathrm{~mm}(\mathbf{a})$ and $12 \mathrm{~mm}$ (b)

indicating the chaotic and regular (periodic) solutions, respectively.

A particular advantage of the ' $0-1$ test' over the frequency spectrum is that it, similarly to the Lyapunov exponent, provides information regarding the dynamics in a single parameter value. Unfortunately, the Lyapunov exponent $[34,35]$ cannot be easily estimated for a non-smooth system. The present system (Fig. 1) used is highly nonlinear with double well potential bi-stability and impacts. Therefore the $0-1$ test can provide the suitable algorithm to identify the chaotic solution [36-39].

Starting from the measured angular output $\alpha(i)$, for sampling points $i=1, \ldots, N_{t}$, (where $\left.N_{t} \approx 400\right)$ we define new coordinates $p(n)$ and $q(n)$ as 
(a)

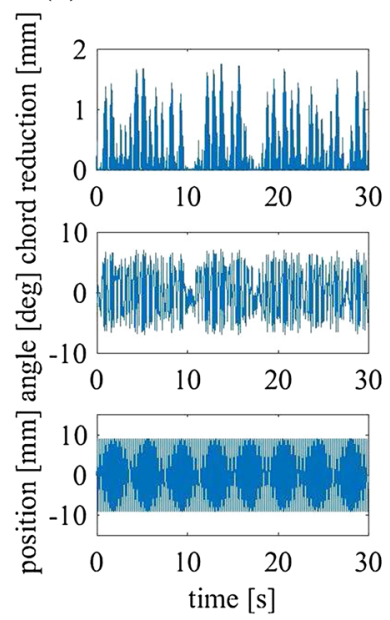

(b)

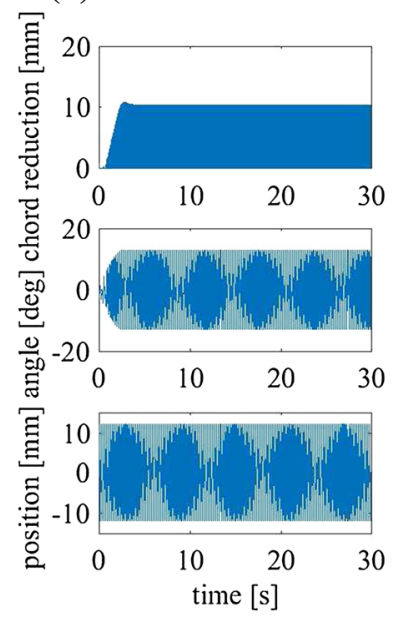

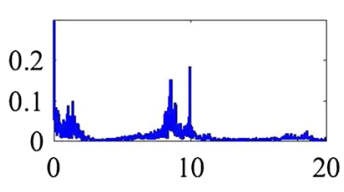
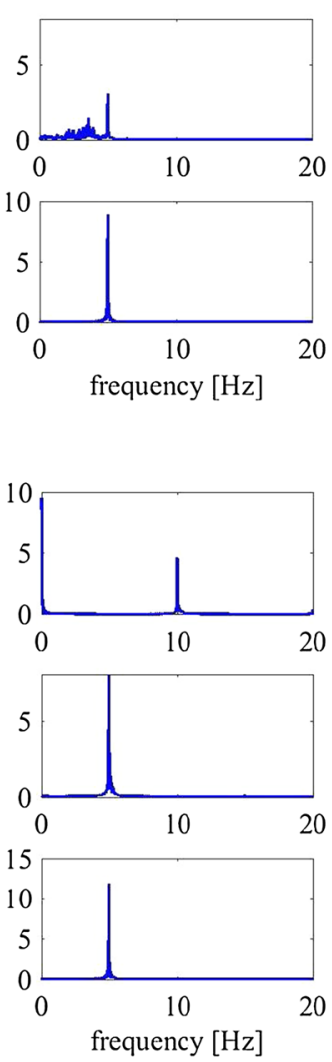

Fig. 5 Simulation results, the frequency and amplitude of kinematic excitation used: $f=5 \mathrm{~Hz}, A=9 \mathrm{~mm}$ (a) and $12 \mathrm{~mm}$ (b)

$$
\begin{aligned}
& p(n)=\sum_{j=0}^{n} \frac{(\alpha(j)-\bar{\alpha})}{\sigma_{\alpha}} \cos (j c), \\
& q(n)=\sum_{j=0}^{n} \frac{\alpha(j)-\bar{\alpha})}{\sigma_{\alpha)}} \sin (j c),
\end{aligned}
$$

where $\bar{\alpha}$ denotes the average value of holder angle $\alpha$ and $\sigma_{\alpha}$ indicates its standard deviation. The parameter $c$ is a constant chosen randomly from the interval $<0, \pi>$. Note that $q(n)$ is a complementary coordinate in the two dimensional space. Furthermore, starting from bounded coordinate $u(i)$ we build a new series of $p(n)$ which can be either bounded or

unbounded depending on dynamics of the examined process.

Continuing the calculation procedure, the total mean square displacement is defined as

$$
\begin{aligned}
M_{c}(n)= & \lim _{N \rightarrow \infty} \frac{1}{N} \sum_{j=1}^{N}\left[(p(j+n)-p(j))^{2}\right. \\
& \left.+(q(j+n)-q(j))^{2}\right],
\end{aligned}
$$

For large enough $n$ the asymptotic growth of $M_{c}(n)$ can be easily characterized by the corresponding ratio $K_{c}(n)$

$$
K_{c}(n)=\frac{\ln (M(n))}{\ln n} .
$$

In the limit of a long time $n<<1$ we obtain the corresponding values of $K_{c}$ for a chosen $c$ value. Note that the $n_{\max }$ and $N$ limits proposed by Gottwald and Melbourne [46-48] $N, n_{\max } \rightarrow \infty$ but simultaneously $n_{\text {max }}$ should be about $N / 10$. In our case $N \approx 400$ and $n_{\max } \approx 10$. These are fairly small numbers, however Fig. 6 shows clearly that they are large enough to identify the periodic and chaotic motions. The estimations are provided for the data of the holder angle from the four selected cases of Figs. 3-5 and presented in Fig. 6a-d respectively. To reduce the calculation time we used local extrema points (similarly to ref. [39]). They are presented in Fig. 6a-d in the upper panels. In the lower panes of the same figures (Fig. 6a-d) we plotted estimated values of $K_{c}$ for 100 randomly sampled values of the parameter $c$. Note that for Fig. 6a, c the median of $K_{c}$ is $K=0.70$ and $K=0.67$ while for Fig. $6 \mathrm{~b} K=0.13$. Results for all the cases are summarized in Table 2.

As the $K$ values are closer to 1 and 0 they indicate strongly that the examined solutions with excitation $f=8 \mathrm{~Hz}$ and $A=12 \mathrm{~mm}$ have a periodic nature while for $f=5$ and $8 \mathrm{~Hz}$ and $\mathrm{A}=9 \mathrm{~mm}$ have a chaotic nature. Here, for simplicity, we used whole time series for analysis. Note that in all cases the initial conditions were fixed the pendulum was lasting in one of the potential well with the positive holder angle.

\subsection{Application of a phase synchronization test}

Another important indicator of the dynamical system is a phase synchronization test [40]. It can be easily implemented to the measured or simulated data. 
(a)
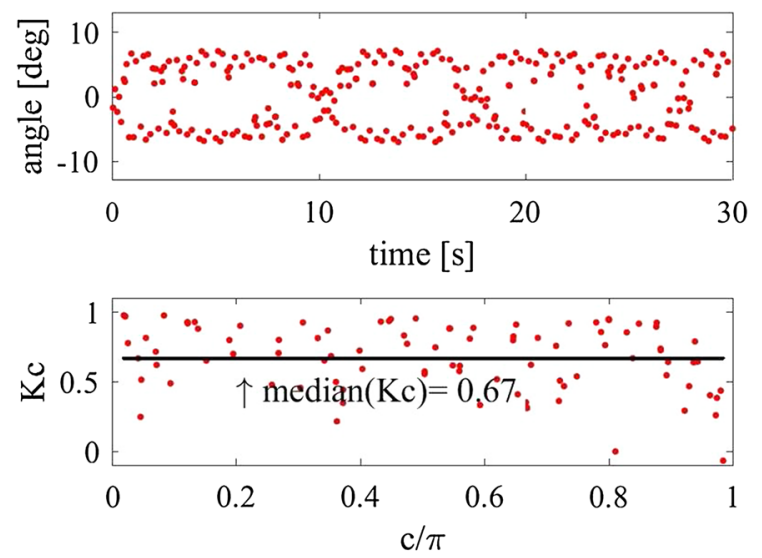

(b)
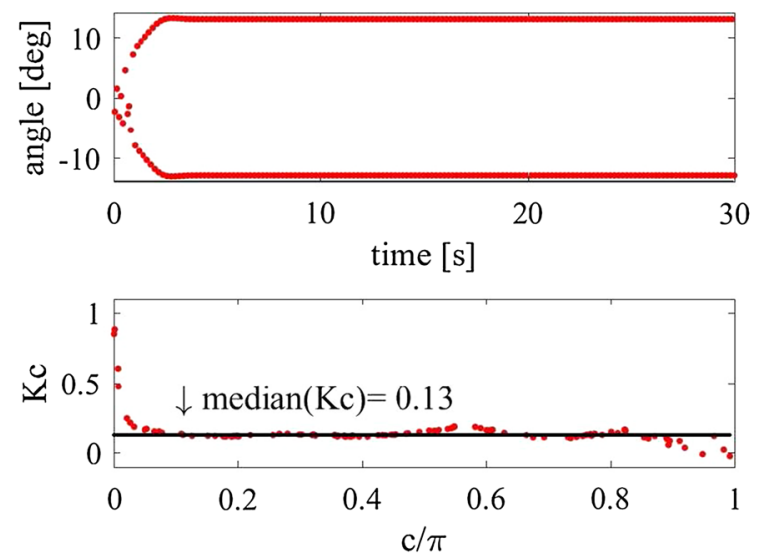

(c)
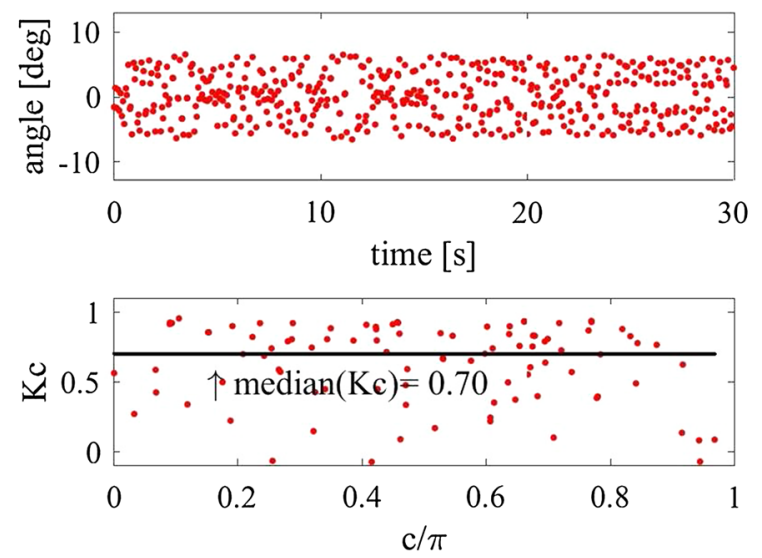

(d)
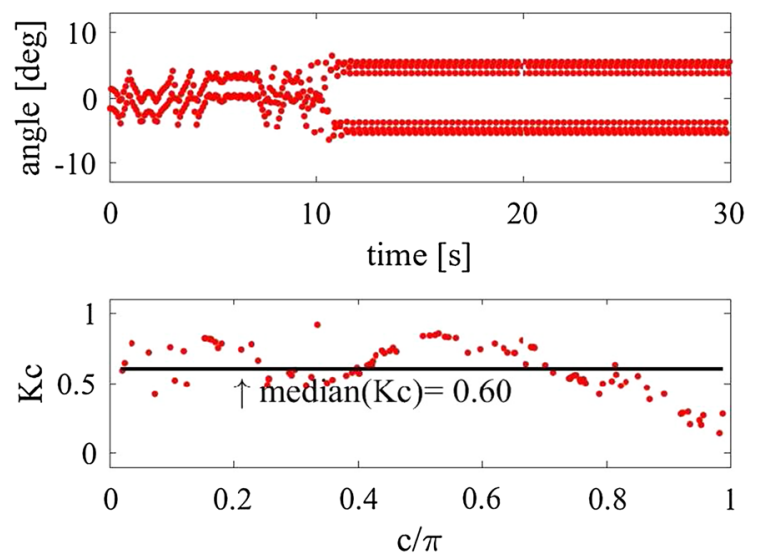

Fig. 6 a-d shows local extrema series (upper panels) collected from the holder angle plots (from Fig. 5a) with $f=5 \mathrm{~Hz}$ and $A=$ $9 \mathrm{~mm}$, (from Fig. 5b) with $f=5 \mathrm{~Hz}$ and $A=12 \mathrm{~mm}$ (from Fig. 4a) with $f=8 \mathrm{~Hz}$ and $A=9 \mathrm{~mm}$, and (from Fig. $3 \mathrm{a}$ ) with $f=5 \mathrm{~Hz}$ and $A=9 \mathrm{~mm}$, respectively. $K_{c}$ (lower panels) were estimated for 100 random values of $c \in\langle 0, \pi\rangle$, while the full lines denote the corresponding median values of $K_{c}: K=\operatorname{median}\left(K_{c}\right)$,

Assuming that we have a two simultaneously sampled time series $x_{1}$ and $x_{2}$ we calculate the corresponding phases $\phi_{1}$ and $\phi_{2}$ along the time evolution by using a Hilbert transform [41].

Their difference

$$
\Delta \phi(k)=\phi_{2}(k)-\phi_{1}(k),
$$

where $k$ denotes the sampling instant, give some statistics about the synchronization of both initial signals. $\Delta \phi$ is defined in the interval $<-\pi / 2, \pi / 2>$. The results in the form of histograms for the four cases studied in Fig. 6 are presented in Fig. 7. Note the single phase for single frequency response of the excitation with $f=5 \mathrm{~Hz}$ and $A=12 \mathrm{~mm}$ (Fig. 6b). The case with excitation of $f=5 \mathrm{~Hz}$ and $A=9 \mathrm{~mm}$ (Fig. 6a) has also nontrivial maximum in its distribution. The other cases (Fig. 6c, d) have a flat angular difference distribution.

Finally, one can calculate the time average

$$
R=\left|\frac{1}{N} \sum_{k=1} \exp (i \Delta \phi(k))\right|,
$$

where $N$ is the number of $\Delta \phi(k)$ series elements. This parameter is an indicator of phase synchronization which having values in the interval $<0,1\rangle$. Value 
Table 2 Results of the $0-1$ test by the parameter $K$ and the phase synchronization test by the parameter $R$ (position of the carriage and holder angle synchronization values)

\begin{tabular}{lcll}
\hline $\begin{array}{l}\text { Amplitude } \\
A(\mathrm{~mm})\end{array}$ & $\begin{array}{l}\text { Frequency } \\
f(\mathrm{~Hz})\end{array}$ & $\begin{array}{l}\mathrm{K}(0-1 \\
\text { test })\end{array}$ & $\begin{array}{l}\mathrm{R} \text { (phase } \\
\text { synchronization) }\end{array}$ \\
\hline 9 & 5 & 0.67 & 0.74 \\
9 & 8 & 0.70 & 0.64 \\
9 & 10 & 0.60 & 0.64 \\
12 & 5 & 0.13 & 0.88 \\
12 & 8 & 0.59 & 0.66 \\
12 & 10 & 0.00 & 0.66 \\
\hline
\end{tabular}

(a)

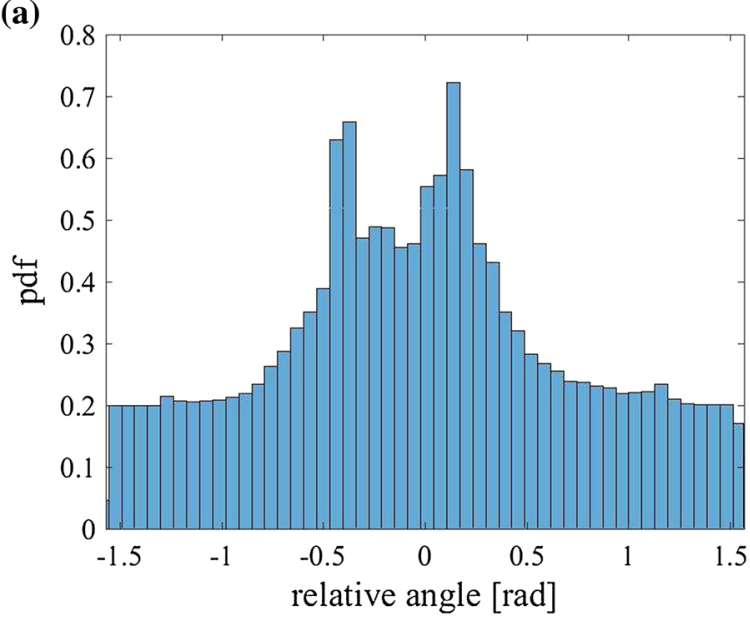

(b)

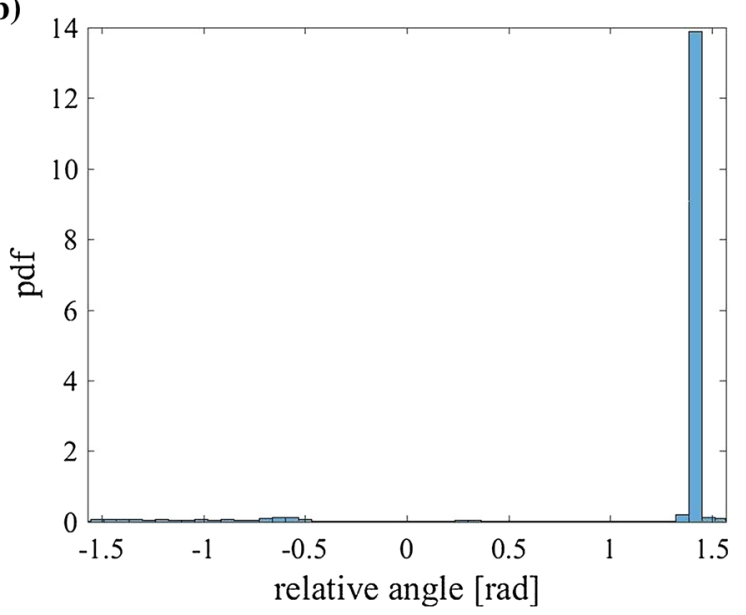

close to 1 states for complete synchronization, while value close to 0 indicates a complete anti-synchronization. For series of uncorrelated random numbers (with uniform distribution) of $\Delta \phi(k) \in[-\pi / 2, p i / 2]$ gives $R=0.64$.

We applied the above test for the considered six cases by substituting series $x_{1}, x_{2}$ by the position of carriage and holder angle. The results are presented in the Table 2. One can see that case $(f=5 \mathrm{~Hz}$ and $A=12 \mathrm{~mm})$ with $R=0.88$ and $(f=5 \mathrm{~Hz}$ and $A=9 \mathrm{~mm}$ ) with $R=0.74$ are characterized by the best phase synchronization. Interestingly, these cases show regular and chaotic solutions, respectively. Other solutions are close to random numbers value. It
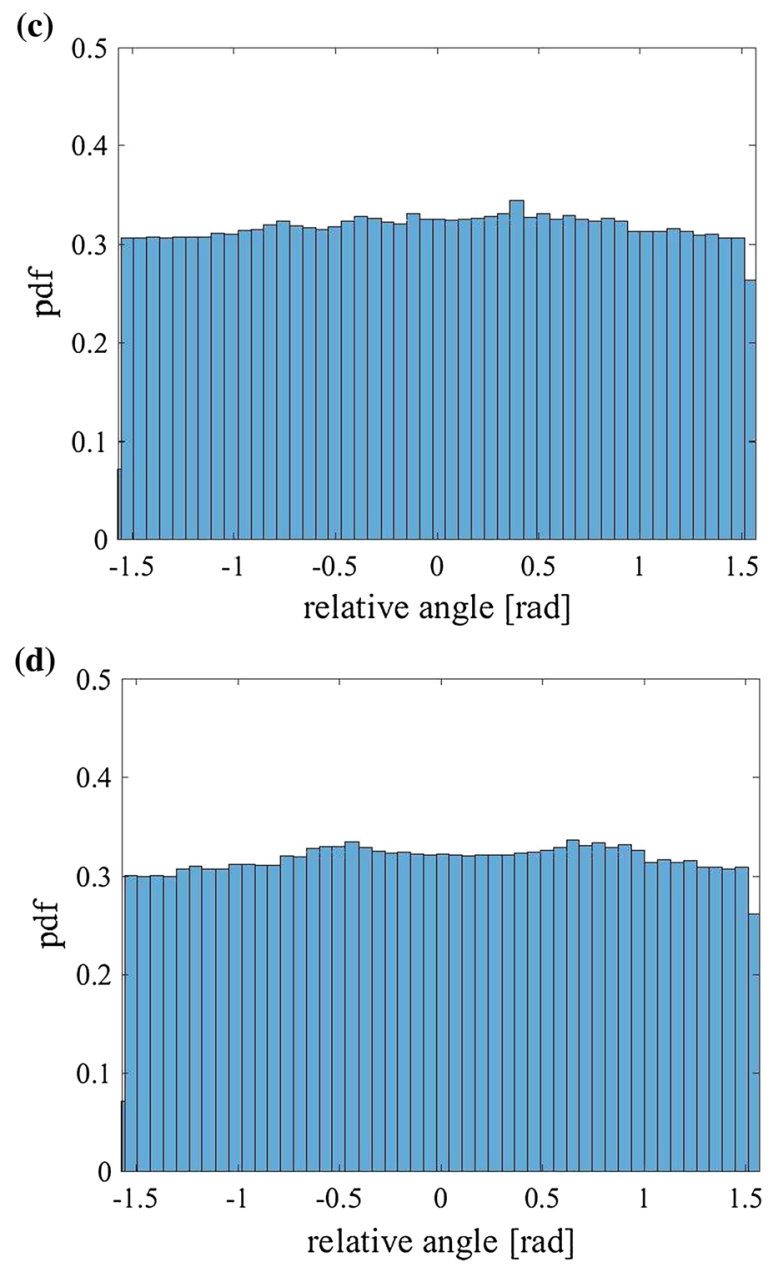

Fig. 7 Histograms (probability density function-pdf) for relative phase differences $\Delta \phi$ for the carriage displacement and holder angle time series calculated for the corresponding cases shown in Figs. 6a-d 
(a)

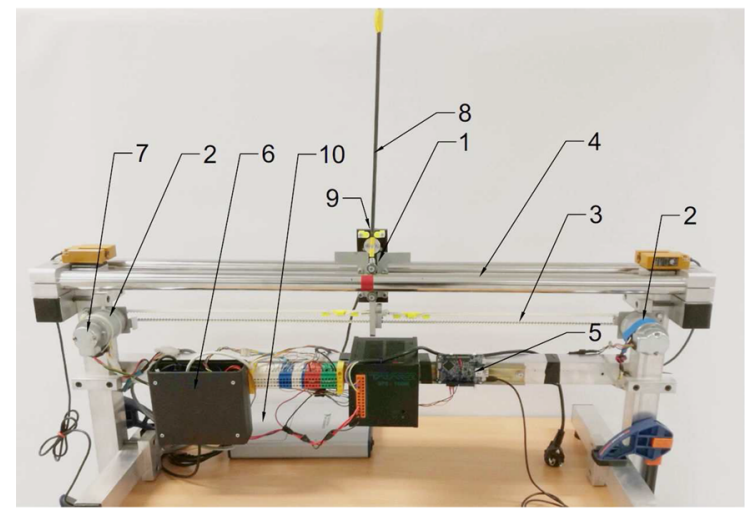

(b)

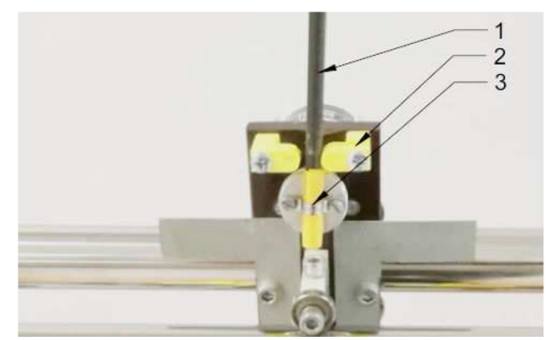

Fig. 8 Conceptual photo of the inverted elastic pendulum with its structure (a), mechanical resonator showing the clearance in rotating beam clamping to the moving base (b)

should be noted that subharmonic solutions evidently destroy the phase synchronization.

\section{Experimental verification}

The experiment was planned using the parameters close to that used in simulations. In the first step we provide a sweep of frequency analysis. In the second step we used the same excitation frequency as in Figs. 3-6. However, for better transparency of the non-periodic solutions we enlarged the excitation amplitudes in cases of $f=5$ and $10 \mathrm{~Hz}$. The displacement of the trolley was measured by an incremental encoder located on the shaft of the driving motor. The accuracy of the measurement depended on the resolution of the incremental encoder as well as the type of decoding of the signal, which in this case was magnified by 4 times. Thanks to that the positioning accuracy of the trolley was $0.173 \mathrm{~mm}$. Similarly, the swing angle was measured (a)
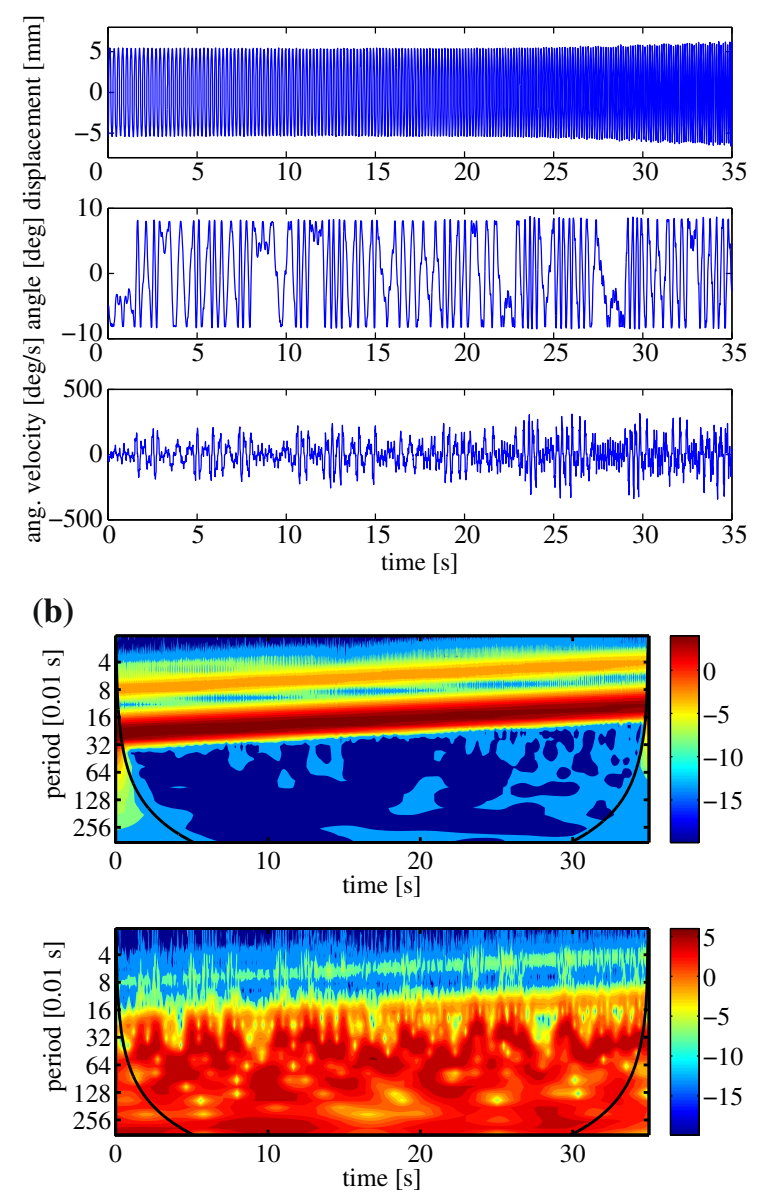

Fig. 9 Frequency sweep smoothly increased [4-8 Hz] during measurements. Starting from upper panel: a the displacement of the base used as excitation, and the system responses as displacement angle and angular velocity. Wavelets power spectrum for the base displacement (excitation) and the angle (response) are in (b). The black contour lines enclose regions of greater than $95 \%$ confidence for a red noise process, and the region below the thin $\mathrm{U}$-shaped curve denotes the cone of influence (COI) below which the results become unreliable [45]

with an incremental encoder in the joint. Encoder resolution as well as signal decoding type made it possible to measure with an accuracy of $0.045^{\circ}$.

For these results we applied the wavelet transform (for a non-stationary course) and Fourier transform (for stationary courses but with the short transients). Finally, to illustrate the synchronization phenomenon of the base and beam motion we show the corresponding phase portraits. 
(a)

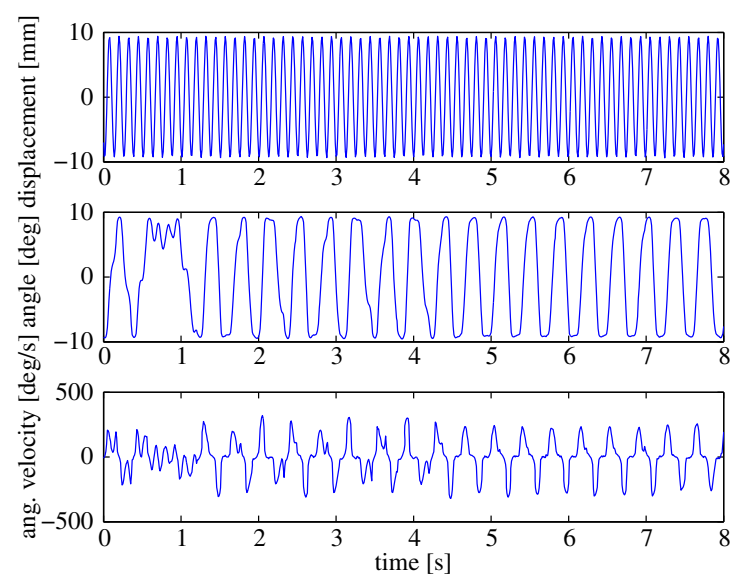

(b)

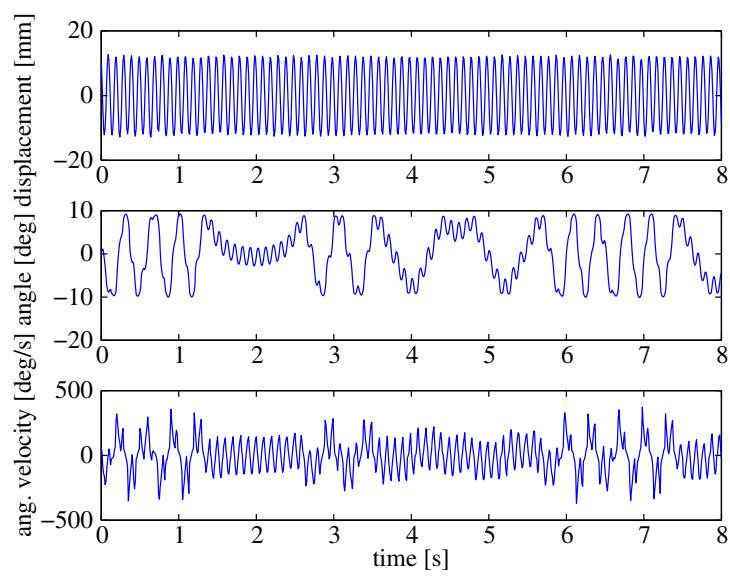

(c)

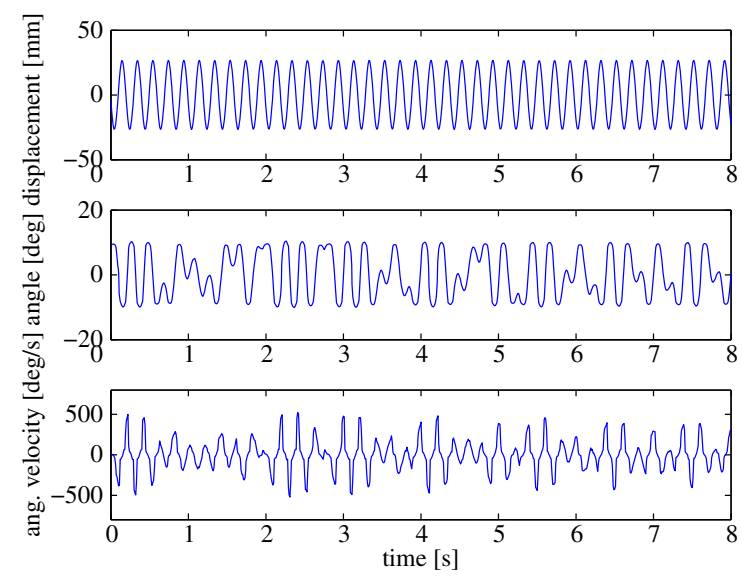

Fig. 10 Displacement of the base used as excitation, and the system responses as displacement angle and angular velocity (from up to down panels, respectively) for three studied cases (frequency and amplitude of excitation: a $8 \mathrm{~Hz}, 9 \mathrm{~mm}$; b $10 \mathrm{~Hz}$, $12 \mathrm{~mm}$ c $5 \mathrm{~Hz}, 25 \mathrm{~mm}$ ). The pendulum amplitude limiter was fixed on $20^{\circ}$ (a)
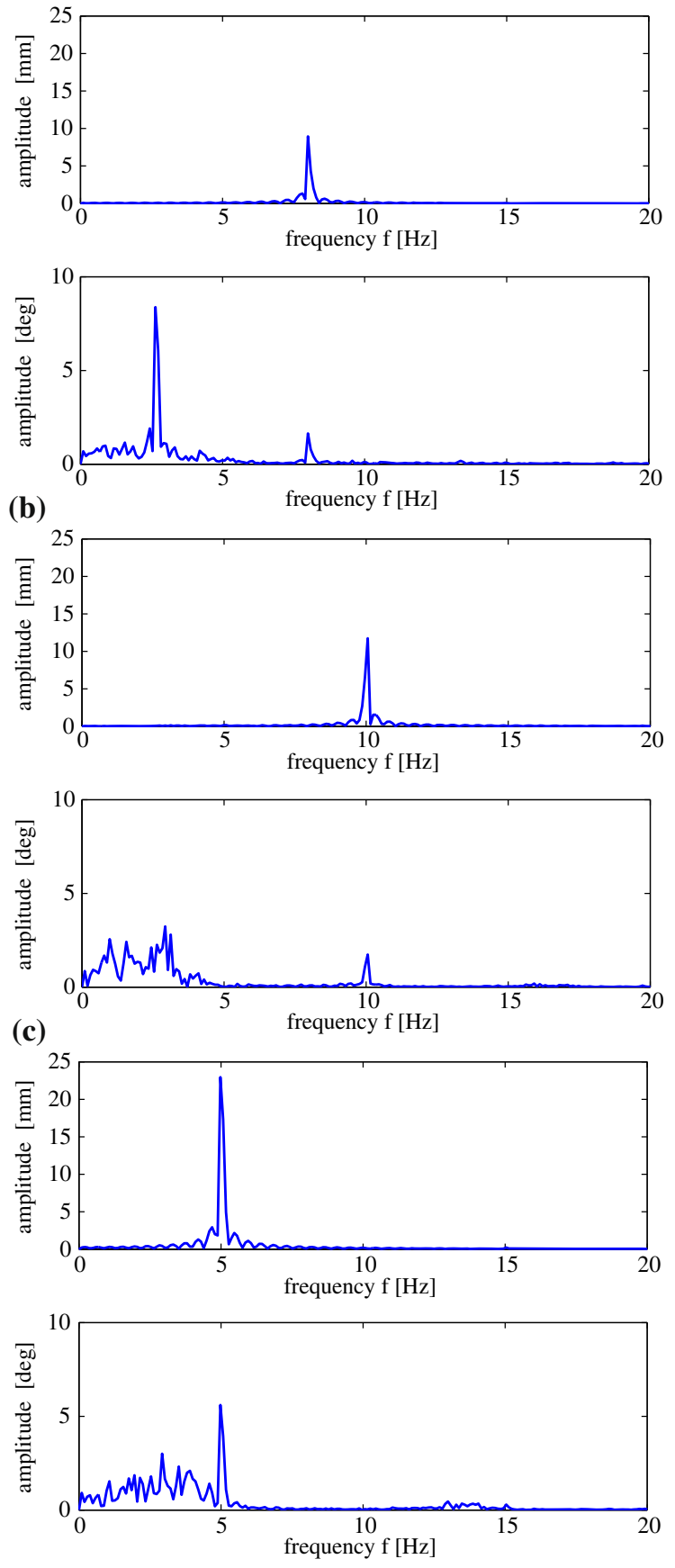

Fig. 11 Fourier spectra of displacement of the base used as excitation, and the system responses as displacement angle and angular velocity (from upper to lower panels respectively) for three studied cases $(\mathbf{a}-\mathbf{c})$ as in Fig. 10 

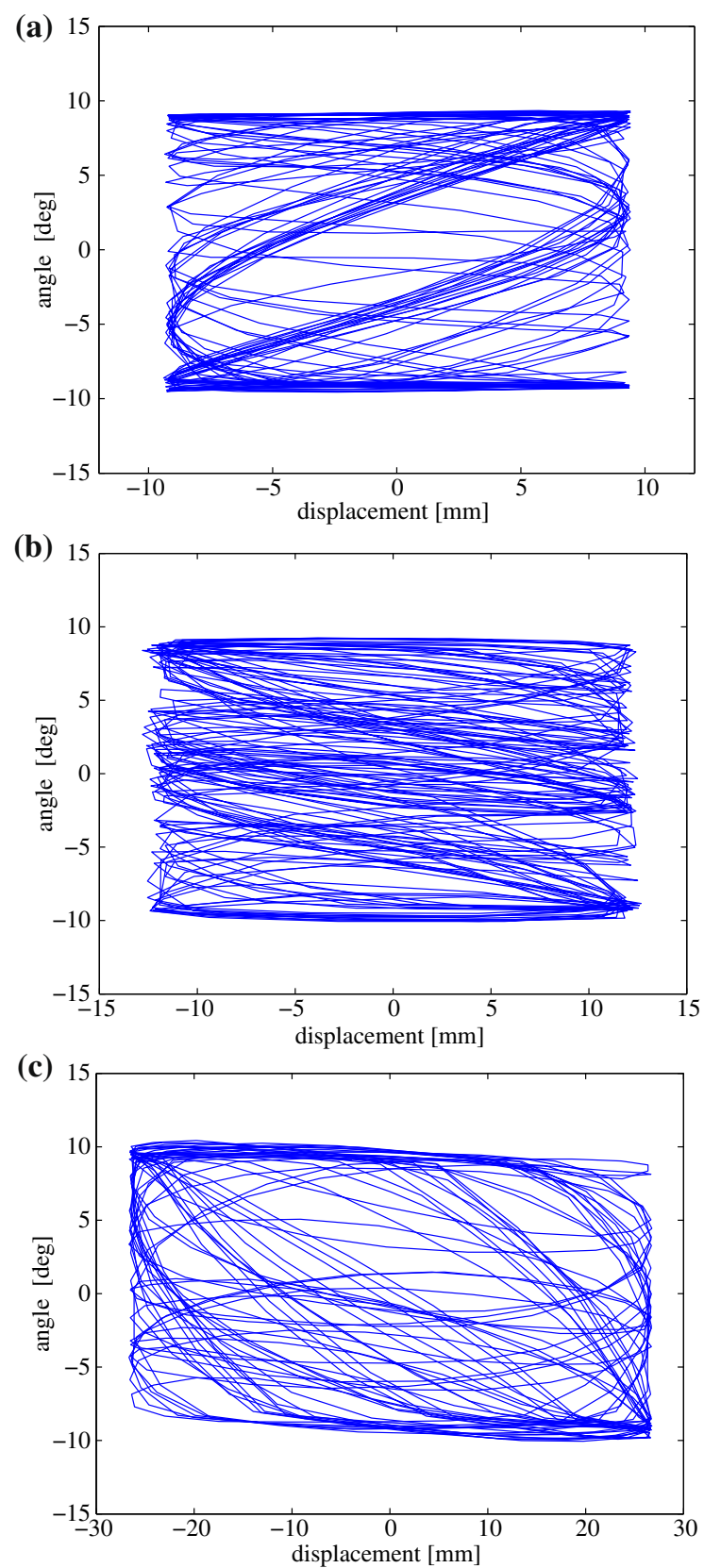

Fig. 12 System responses as displacement angle versus displacement of the base used as excitation, and the system responses as displacement angle (from up to down panels respectively) for three studied cases $(\mathbf{a}-\mathbf{c})$ as in Fig. 9

\subsection{Experimental stand}

The experimental stand for an inverted pendulum is presented in Fig. 8. The scheme of Fig. 8a shows the carriage of the inverted pendulum (1), driven by the
DC motor (2) via a belt drive (3) and moved along the guides (4). Motion of the carriage is performed by generating step/dir signals; by a micro-controller (5); on the inputs of a servo controller (6). The servo controller uses an incremental encoder (7) and closed-loop PID controller to stabilize the setpoint of movement and reflecting the generated trajectory. Movement of the carriage causes oscillation of the inverted pendulum (8) which is recorded using an incremental encoder (9) which is simultaneously a pivot of the pendulum. The pendulum has a limited range of motion, through the use of configurable position buffers. Measuring the position of the carriage and the angle of the pendulum is performed with the help of counter inputs of a data acquisition card NI USB-6341 (10). The acquisition takes place at $100 \mathrm{kHz}$. An important element of the stand is a micro-controller which generates the waveforms of the harmonic movement of variable frequency. This enables a smooth transition through the resonance frequencies of the system. Fig. $8 \mathrm{~b}$ presents the (1) pendulum, (2) bumpers, (3) revolute joint.

This research setup was designed to generate harmonic excitations for an inverted pendulum with stoppers.

\subsection{Results and discussion}

By using the above presented experimental setup (Figs. 1, 7 and Table 1) we performed a series of experiments for the following parameters: masses of the elastic beam, $m=0.055 \mathrm{~kg}$, and tip mas, $M=0.012 \mathrm{~kg}$, characteristic lengths, $l=375 \mathrm{~mm}$, $d=3.5 \mathrm{~mm}, D=6.0 \mathrm{~mm}$. The results of the angular response at the hinged joint (beam holder) under the assumed horizontal excitation are presented in the Figs. 9-11.

Figure 9 shows the results obtained in incremental frequency sweep (4-8 Hz) during measurements. Starting from upper panel we show in (a) the displacement of the base used as excitation, and the system responses as displacement angle and angular velocity. The corresponding wavelet power spectra for the base displacement (excitation) and the angle (response) are in (b). Here, the wavelets of base displacement show the basic frequency systematically accompanied by lower periods. This is the effect of imperfectness in respect to harmonic local fitting. 
Here we performed a wavelet analysis. This analysis is very useful for non-stationary time series $[42,43]$. Basing on the measured value time series $x$ ( $t$ ) (indicating the base displacement or the holder angular response) we applied the continuous wavelet transform (CWT) [44, 45]:

$$
W_{s, n}(x)=\sum_{i=1}^{N} \frac{1}{s} \psi\left(\frac{i-n}{s}\right) \frac{\left(x\left(t_{i}\right)-<x>\right)}{\sigma_{x}},
$$

where $\psi(t)$ is wavelet function, $\left\langle x>\right.$ and $\sigma_{x}$ are the averages and standard deviations of acceleration, the letters $s$ and $n$ denote the scale and the time indices, respectively. The square modulus of the CWT defines the wavelet power spectrum (WPS), $P_{W}$, of the corresponding time series $x(t)$ :

$$
P_{W}=\left|W_{s, n}\right|^{2} \text {. }
$$

A complex Morlet type was used as the mother wavelet $\psi(t)$. It consists of a plane wave modulated by a Gaussian function:

$$
\psi(\eta)=\pi^{-1 / 4} \mathrm{e}^{\mathrm{i} \theta_{0} \eta} \mathrm{e}^{-\eta^{2} / 2},
$$

where $\theta_{0}$ is the center frequency. It is also referred to as the order of the wavelet and $\eta$ is a renormalized time variable. To balance the time/frequency resolutions we adopted $\theta_{0}=6$, which defines six oscillations in the mother wavelet. A detailed discussion on wavelets can be found in [44, 45].

Figure 9a, in the upper panel, shows the dominating frequency linear increase as was expected for the smoothly increased quasi harmonic excitation. Consequently, the wavelets of angular response (Fig. 9b bottom panel) confirm its complex character in contrast to the very regular base variations (Fig. 9b upper panel). The angular response indicates a number of shorter periodics in the region 0.32 $0.64 \mathrm{~s}$. Due to nonlinearities the angular response of the resonator is not synchronized with the excitation signal. This is clearly visible in the wavelets. It is worth to note that we observed some differences between increasing and decreasing way of frequency scanning however there was a similarity in the general intermittent like response.

Using the stationary conditions can shed more light on solution cases. In Fig. 10a-c we show base displacement, and angular response (angle and angular velocity) for selected cases. The analysed cases correspond to the simulated results (Figs. 3-6), however the amplitudes are different to express the mutiscale character of the system responses in the case of non-periodic vibrations. The corresponding Fourier spectra for base and pendulum angular displacements are presented in Fig. 11a-c. One can identify some interesting effects on these images. Firstly, in Figs. 10a and 11a one can observe the frequency dividing from $8 \mathrm{~Hz}$ down to about $2.6 \mathrm{~Hz}$ (one third). This is a nonlinear effect suggesting a subharmonic solution. Secondly, all of cases do not present periodic responses with respect to angular motion of inverted pendulum, in spite of the periodic character of base excitation. This is due to the intermittency phenomenon which enables the system to switch between various nonlinear solutions. Consequently Figs. 10b, c and 11b, c show the typical band response in place of the frequency peaks appearing for a periodic response.

More transparent identification of intermittency and synchronization phenomenon can be based on the phase portraits in Fig. 12. If we define the system more generally, the synchronization phenomenon would correspond to single paths in the portraits. Consequently, Fig. 12b appears to be less synchronized compared to Fig. 12a, c. This confirms the results in the Fourier spectra (see Fig. $11 \mathrm{~b}$ and Figs. 11a, c, respectively). In the context of phase synchronization the clarification would differ as the leading frequency should be the same as in the carriage position excitation. Therefore the case in Fig. 12c correspond to the best synchronization $(R=0.64)$. For other cases we obtained $R=0.65$ for the case in Fig. $12 \mathrm{~b}$ and $R=0.64$ for the case in Fig. 12a, respectively.

\section{Conclusions}

We presented preliminary results of an elastic inverted pendulum with amplitude limiters excited horizontally. This model was motivated by energy harvesting systems where the nonlinear mechanical resonator should have a broad energy transfer. We explore theoretically and experimentally the complex responses of this system for the fixed value of amplitude clearance (imperfect clamping) excited by stationary and non-stationary horizontal excitations. 
The results indicate that such a resonator shows the broadband frequency response and can be successfully adjusted to energy harvesting. The system shows also a capability of decreasing or increasing the particular input frequency including non-periodic (complex) broadband output. Presumably, this is due to the intermittent passing through the potential barrier at the vicinity of the internal subharmonic resonance of the inverted pendulum swinging oscillation with additional bending of the beam.

Note that mechanical damping is an important issue in this consideration. It has a considerable effect on reduction of free vibration frequency components of the beam and tip mass system. However mechanical damping should not be decreased in the process of energy harvesting because of two reasons. Firstly, damping is broadening the frequency response band, which a beneficial factor for ambient sources with variable frequency and amplitude. Consequently, the optimum conditions of energy harvesting (which were developed for the linear system but can be approximately applied to nonlinear systems) are governed by, so called, impedance matching [5, 6]. In our simplified model the most mechanical dissipation is present during impacts. It could also arise in the rotating handle of the beam hinged connection and from internal damping of the beam material and aerodynamic losses of beam in motion in air. The broadband frequency effect is realized by off resonance work. This effect is visible in the experimental frequency sweep (Fig. 9) where for the large amplitude response is present for the most of studied frequency range.

Acknowledgements The research was financed in the framework of the project Lublin University of TechnologyRegional Excellence Initiative, funded by the Polish Ministry of Science and Higher Education (Contract No. 030/RID/2018/ 19).

\section{Compliance with ethical standards}

Conflict of interest The authors declare that they have no conflict of interest.

Open Access This article is distributed under the terms of the Creative Commons Attribution 4.0 International License (http://creativecommons.org/licenses/by/4.0/), which permits unrestricted use, distribution, and reproduction in any medium, provided you give appropriate credit to the original author(s) and the source, provide a link to the Creative Commons license, and indicate if changes were made.

\section{References}

1. Mitcheson PD, Yeatman EM, Rao GK, Holmes AS, Green TC (2008) Energy harvesting from human and machine motion for wireless electronic devices. Proc IEEE 96:1457-1486

2. Harne RL, Wang KW (2013) A review of the recent research on vibration energy harvesting via bistable systems. Smart Mater Struct 22:023001

3. Pellegrini SP, Tolou N, Schenk M, Herder JL (2013) Bistable vibration energy harvesters: a review. J Intell Mater Syst Struct 24:1303-1312

4. Twiefel J, Westermann H (2013) Survey on broadband techniques for vibration energy harvesting. J Intell Mater Syst Struct 24:1291-1302

5. Beeby SP, Tudor MJ, White NM (2006) Energy harvesting vibration sources for microsystems applications. Meas Sci Technol 17:R175-R195

6. Koszewnik A, Grzes P, Walendziuk W (2015) Mechanical and electrical impedance matching in a piezoelectric beam for energy harvesting. Eur Phys J Spec Top 224:27192731

7. Badel A, Guyomar D, Lefeuvre E, Richard C (2006) Piezoelectric energy harvesting using a synchronized switch technique. J Intell Mater Syst Struct 17:831-839

8. Lefeuvre E, Badel A, Richard C, Petit L, Guyomar DA (2006) Comparison between several vibration-powered piezoelectric generators for standalone systems. Sens Actuat A Phys 126:405-416

9. Daqaq MF, Masana R, Erturk A, Quinn DD (2014) On the role of nonlinearities in vibratory energy harvesting: a critical review and discussion. Appl Mech Rev 66:040801

10. Erturk A, Hoffmann J, Inman DJ (2009) A piezomagnetoelastic structure for broadband vibration energy harvesting. Appl Phys Lett 94:254102

11. Cottone F, Vocca H, Gammaitoni L (2009) Nonlinear energy harvesting. Phys Rev Lett 102:080601

12. Syta A, Litak G, Friswell MI, Adhikari S (2016) Multiple solutions and corresponding power output of a nonlinear bistable piezoelectric energy harvester. Eur Phys J B 89:99

13. Huguet T, Badel A, Lallart M (2017) Exploiting bistable oscillator subharmonics for magnified broadband vibration energy harvesting. Appl Phys Lett 111:173905

14. Huguet T, Badel A, Druet O, Lallart M (2018) Drastic bandwidth enhancement of bistable energy harvesters: study of subharmonic behaviors and their stability robustness. Appl Energy 226:607-617

15. Vocca H, Neri I, Travasso F, Gammaitoni L (2012) Kinetic energy harvesting with bistable oscillators. Appl Energy 97:771-776

16. Lin J-T, Walsh K, Alphenaar B (2012) Enhanced stochastic, subharmonic, and ultraharmonic energy harvesting. J Intell Mater Syst Struct 24:1324-1331

17. Rocha RT, Balthazar JM, Tusset AM, Piccirillo V, Felix JLP (2017) Nonlinear piezoelectric vibration energy harvesting from a portal frame with two-to-one internal resonance. Meccanica 52:2583-2602

18. Iliuk I, Balthazar JM, Tusset AM, Piqueira JRC, de Pontes BR, Felix JLP, Bueno AM (2014) Application of passive control to energy harvester efficiency using a nonideal 
portal frame structural support system. J Intell Mater Syst Struct 25:417-429

19. Friswell MI, Ali SF, Adhikari S, Lees AW, Bilgen O, Litak G (2012) Nonlinear piezoelectric vibration energy harvesting from a vertical cantilever beam with tip mass. J Intell Mater Syst Struct 23:1505-1521

20. Litak G, Friswell MI, Adhikari S (2016) Regular and chaotic vibration in a piezoelectric energy harvester. Meccanica 51:1017-1025

21. Litak G, Rysak A, Borowiec M, Scheffler M, Gier J (2016) Vertical beam modal response in a broadband energy harvester. Proc Inst Mech Eng Part K J Multi Body Dyn 230:541-552

22. Harris P, Bowen CR, Kim HA, Litak G (2016) Dynamics of a vibrational energy harvester with a bistable beam: voltage response identification by multiscale entropy and "0-1" test. Eur Phys J Plus 131:109

23. Stoykov S, Manoach E, Litak G (2015) Vibration energy harvesting by a Timoshenko beam model and piezoelectric transducer. Eur Phys J Spec Top 224:2755-2770

24. Tang J, Ren G (2009) Modeling and simulation of a flexible inverted pendulum system. Tsinghua Sci Technol 14:22-26

25. Xu C, Yu X (2014) Mathematical modeling of elastic inverted pendulum control system. J Control Theory Appl 3:281-282

26. Semenov ME, Meleshenko PA, Solovyov AM, Semenov AM (2015) Hysteretic nonlinearity in inverted pendulum problem. In: Moohamed B (ed) Structural nonlinear dynamics and diagnosis. Springer, Cham, pp 463-506

27. Semenov ME, Solovyev AM (2016) Stabilization of elastic pendulum with hysteresis. In: International conference information technology and nanotechnology-Samara 2016 CEUR workshop proceedings, Samara, pp 650-657. https://doi.org/10.18287/1613-0073-2016-1638-650-657

28. Semenov ME, Solovyov AM, Meleshenko PA (2015) Elastic inverted pendulum with backlash in suspension: stabilization problem. Nonlinear Dyn 82:677-688

29. Wolszczak P, Lygas K, Litak G (2018) Dynamics identification of a piezoelectric vibrational energy harvester by image analysis with a high speed camera. Mech Syst Sign Process 107:43-52

30. Gottwald GA, Melbourne I (2004) A new test for chaos in deterministic systems. Proc R Soc Lond A 460:603-611

31. Gottwald GA, Melbourne I (2005) Testing for chaos in deterministic systems with noise. Phys D 212:100-110

32. Bernardini D, Litak G (2016) An overview of 0-1 test for chaos. J Braz Soc Mech Sci Eng 38:1433-1450

33. Gottwald GA, Melbourne I (2016) The 0-1 test for chaos: a review. In: Skokos CH, Gotwald GA, Laskar J (eds) Chaos detection and predictability. Springer, Heidelberg, pp 221247

34. Wolf A, Swift JB, Swinney HL, Vastano JA (1985) Determining Lyapunov exponents from a time series. Phys D 16:285-317

35. Kantz H, Schreiber $T$ (1997) $T$ non-linear time series analysis. Cambridge University Press, Cambridge

36. Litak G, Syta A, Wiercigroch M (2009) Identification of chaos in a cutting process by the $0-1$ test. Chaos Solitons Fractals 40:2095-2101

37. Litak G, Schubert S, Radons G (2012) Nonlinear dynamics of a regenerative cutting process. Nonlinear Dyn 69:1255-1262

38. Krese B, Govekar E (2012) Nonlinear analysis of laser droplet generation by means of $0-1$ test for chaos. Nonlinear Dyn 67:2101-2109

39. Litak G, Bernardini D, Syta A, Rega G, Rysak A (2013) Analysis of chaotic non-isothermal solutions of thermomechanical shape memory oscillators. Eur Phys J Spec Top 222:1637-1647

40. Pikovsky A, Rosenblum M, Kurths J (2001) Synchronization, a universal copncept in nonlinear sciences. Cambridge University Press, Cambridge

41. Hilbert D (1953) Grundzüge einer allgemeinen theorie der linearen integralgleichungen. Chelsea Pub. Co., White River Junction

42. Borowiec M, Sen AK, Litak G, Hunicz J, Koszalka G, Niewczas A (2010) Vibrations of a vehicle excited by real road profiles. Forsch Ing 74:99-109

43. Lonkwic P, Lygas K, Wolszczak P, Molski S, Litak G (2017) Braking deceleration variability of progressive safety gears using statistical and wavelet analyses. Measurment 110:90-97

44. Kumar P, Foufoula-Georgiou E (1997) Wavelet analysis for geophysical applications. Rev Geophys 35:385-412

45. Torrence C, Compo GP (1998) A practical guide to wavelet analysis. Bull Am Meteorol Soc 79:61-78

46. Gottwald GA, Melbourne I (2009) On the implementation of the 0-1 test for chaos. SIAM J Appl Dyn Syst 8:129-145

47. Melbourne I, Gottwald GA (2008) Power spectra for deterministic chaotic dynamical systems. Nonlinearity 21:179-189

48. Gottwald GA, Melbourne I (2009) On the validity of the 01 test for chaos. Nonlinearity 22:1367-1382

Publisher's Note Springer Nature remains neutral with regard to jurisdictional claims in published maps and institutional affiliations. 